DOI: $10.15421 / 4220005$

УДК 539.3

А. А. Дисковский, д-р техн. наук,

А. А. Косиченко, канд. техн. наук, А. В. Махницкий

\title{
УСТОЙЧИВОСТЬ ГОФРИРОВАННОГО КОЛЬЦА ПОД ДЕЙСТВИЕМ ВНЕШНЕГО ДАВЛЕНИЯ
}

Рассматривается задача устойчивости гофрированного кольца при гидростатическом давлении. Анализируются два основных подхода к решению: метод эквивалентной жесткости и метод асимптотической гомогенизации. Применение метода асимптотической гомогенизации позволило математически обосновать и уточнить метод эквивалентной жесткости. Проведен анализ влияния параметров гофрировки на величину критического давления.

Ключевые слова: устойчивость; гофрированное кольцо; внешнее давление; эквивалентная жесткость; асимптотическая гомогенизация.

Гофрирование гладких оболочек часто используют для изменения их жесткостей. В частности, для цилиндрических оболочек при внешнем давлении применяется гофрирование образующей, гофрирование направляющей значительно повышает устойчивость таких оболочек при осевом сжатии. Поэтому исследование устойчивости гофрированных оболочек при внешнем давлении имеет практическое значение.

Методики, применяемые в настоящее время для расчета гофрированных пластин и оболочек, можно разделить на три основные группы: компьютерные модели, основанные на методе конечных элементов, метод эквивалентной изгибной жесткости, и метод асимптотической гомогенизации. Метод конечных элементов позволяет рассчитывать практически любую гофрированную оболочку $[1,7]$, но на начальных этапах проектирования полезными являются простые аналитические решения. До развития цифровых технологий основным методом расчета гофрированных оболочек было использование моделей с эквивалентной жесткостью $[2,5]$. Основным недостатком такого подхода является трудность оценки его точности и области применимости. В настоящей работе используется метод асимптотической гомогенизации $[3,4]$.

Постановка задачи. Радиус гофрированного кольца (рис. 1) в полярной системе координат

$$
r=R(1+h g(n \varphi))
$$

() А. А. Дисковский, А. А. Косиченко, А. В. Махницкий, 2020 
где $R$ - радиус базового кругового кольца; $h=\frac{H}{R}, H$ - амплитуда гофра; $g(n \varphi)$ - периодическая функция с периодом $2 \pi / n$, который назовем шагом гофра, $n$ - количество волн гофра; $0 \leq \varphi \leq 2 \pi ;|h g| \leq 1$; $h \ll 1 ; n \gg 1$.

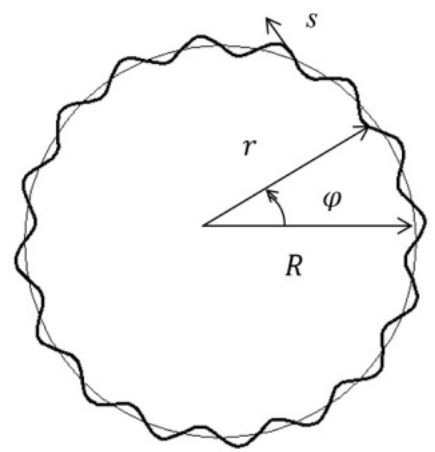

Рис. 1 - Гофрированное кольцо, $h=0,05 ; n=16$.

Будем считать, что для рассматриваемого кольца выполняется гипотеза плоских сечений и величина изгибающего момента пропорциональна изменению кривизны срединной поверхности кольца в рассматриваемом сечении.

В работе изучается общая потеря устойчивости гофрированного кольца при следующих допущениях: не рассматриваются пространственные формы равновесия; при деформации нагрузка остается направленной по нормали к деформированной срединной поверхности кольца и её интенсивность не меняется.

Метод эквивалентной жесткости. Заменим гофрированное кольцо (1) круговым радиуса $R$ с одинаковым поперечным сечением, но с некоторой приведенной изгибной жесткостью. Для определения этой жесткости выделим одну волну гофра, при этом, в силу большого числа таких вол, изменением кривизни равноотстоящей от вершин гофра окружности можно пренебречь. Приведенная изгибная жесткость [5]

$$
D=\epsilon E I
$$

где $E I$ исходная изгибная жесткость кольца; $\epsilon==\frac{1}{2 \pi R} \int_{0}^{2 \pi} A d \xi, \xi=$ $n \varphi ; A=\sqrt{r^{2}+r_{\varphi}^{2}}$.

Критическое давление для кругового кольца заданной изгибной жесткости хорошо известно [7], поэтому для кольца с изгибной жесткостью (2) получаем

$$
p=\epsilon,
$$


где $p=\frac{P}{p^{0}}, P=\frac{3 \epsilon E I}{R^{3}}-$ критическое давление гладкого кругового кольца с жесткостью (2); $p^{0}=\frac{3 E I}{R^{3}}-$ критическое давление кругового кольца с изгибной жесткостью $E I$.

Гофрировка (1) имеет два характерных размера: $n$ и $h$. Критическое давление (3) зависит от параметра $n h$, который можно назвать параметром гофрировки. Для $h=0$ имеем $\epsilon=1$, с ростом $n h$ критическое давление падает (рис. 2).

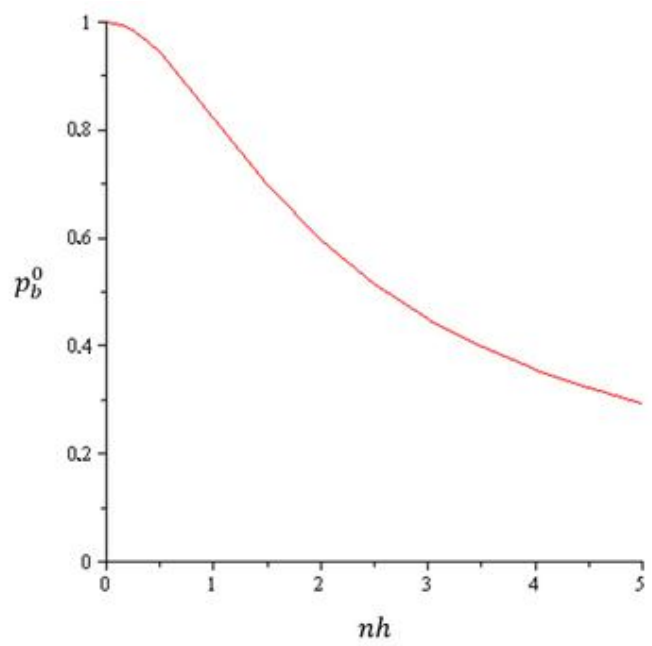

Рис. 2 - Уменьшение критического давления гофрированного кольца по отношению к критическому давлению кругового кольца $p^{0}$ базового радиуса в зависимости от величины параметра гофрирования $\boldsymbol{n h}(\boldsymbol{h}=0,05 ; \boldsymbol{n}=0 . .100)$

Если же проводить сравнение с гладким кольцом, длина окружности которой равна длине криволинейной оси гофрированного кольца (1), то гофрирование повышает критическое давление

$$
\bar{p}=\epsilon^{-2},
$$

где $\bar{p}=\frac{P}{\bar{P}}, \bar{P}=\frac{3 E I}{\left(\epsilon^{-1} R\right)^{3}}-$ критическое давление гладкого кольца равного периметра.

Сравнение гладкого кольца с гофрированным ( $h=0.05 ; n=16)$ одинакового периметра показало, что критическая нагрузка гофрированного кольца на $31 \%$ больше, но оно ограничивает на 23.6 \% меньшую площадь. Сравнение же с базовым, равноотстоящим от вершин гофра гладким кольцом, дало уменьшение критического давления для гофрированного кольца на $12,6 \%$ и уменьшение ограниченной площади 
на $0,125 \%$. При этом длина дуги гофрированного кольца на $14,5 \%$ больше длины базового.

Метод асимптотической гомогенизации. Запишем уравнения равновесия гофрированного кольца как криволинейного стержня [6] в полярной системе координат (рис. 1)

$$
\begin{gathered}
\frac{1}{A} N^{\prime}{ }_{\varphi}=q_{t^{\prime}}+\frac{Q^{\prime}}{\rho} ; \frac{1}{A} Q^{\prime}{ }_{\varphi}=q_{n^{\prime}}-\frac{N^{\prime}}{\rho} ; \\
\frac{1}{A} M^{\prime}{ }_{\varphi}=Q^{\prime},
\end{gathered}
$$

где $(\cdot)_{\varphi}=\frac{d(\cdot)}{d \varphi} ; \quad N^{\prime}, Q^{\prime}, M^{\prime}-$ внутренние усилия и изгибающий момент; $q_{t^{\prime}}, q_{n^{\prime}}-$ проекции внешней нагрузки на подвижные оси $t^{\prime}, n^{\prime}$.

Введем в рассмотрение функции $N, Q, M, q_{t}, q_{n}$ - проекции внутренних усилий, изгибающего момента и внешней нагрузки на $t, n$ - подвижные оси базового кольца, равноотстоящего от вершин гофра, и выразим через эти проекции внутренние усилия и момент

$$
\begin{gathered}
N^{\prime}=N \sin \theta+Q \cos \theta ; \quad N^{\prime}=N \sin \theta+Q \cos \theta ; \\
M^{\prime}=M-z N ; \\
q_{t}^{\prime}=q_{t} \cos \theta+q_{n} \sin \theta ; \quad q_{n}^{\prime}=q_{n} \cos \theta-q_{t} \sin \theta,
\end{gathered}
$$

где $z=r-R ; \theta-$ угол между векторами $n$ и $t^{\prime} ; \sin \theta=\frac{r}{A} ; \cos \theta=\frac{r_{\varphi}}{A}$.

Подставив выражения (6) в уравнения равновесия (5), можно получить

$$
\begin{gathered}
\frac{1}{A} N_{\varphi}=q_{t}+\frac{Q}{\rho} ; \quad \frac{1}{A} Q_{\varphi}=q_{n}-\frac{N}{\rho} ; \\
M_{\varphi}=z N_{\varphi}+r Q .
\end{gathered}
$$

Физические уравнения криволинейного стержня [6] относительно проекций внутренних усилий, изгибающего момента (7) и проекций перемещений на оси $t, n$ приводятся к виду

$$
\begin{gathered}
r N+r_{\varphi} Q=E F \frac{1}{A}\left(r \varepsilon+r_{\varphi} \vartheta\right) ; \\
M-Z N=E I \frac{1}{A}\left(\frac{1}{A^{2}}\left(r_{\varphi} \varepsilon-r \vartheta\right)\right)_{\varphi}
\end{gathered}
$$

где $E, F, I$ - модуль упругости материала, площадь и момент инерции поперечного сечения кольца соответственно; $\varepsilon=u_{\varphi}+w ; \vartheta=w_{\varphi}-u$.

Ограничимся рассмотрением «общей» потери устойчивости, считая, что при этом базовая ось кольца остается нерастяжимой: $u_{\varphi}+w=0$. Тогда фризические соотношения (8) примут вид

$$
\begin{gathered}
r N+r_{\varphi} Q=E F \frac{r \varphi \vartheta}{A} ; \\
M-Z N=-E I \frac{1}{A}\left(\frac{r \vartheta}{A^{2}}\right)_{\varphi}
\end{gathered}
$$


Для разрешающей системы уравнений (7), (9) применим метод гомогенизации, основанный на асимптотических двух масштабных разложениях

$$
\begin{gathered}
N=\sum_{k=0}^{\infty} n^{-k} N_{k}(\varphi, \xi) ; Q=\sum_{k=0}^{\infty} n^{-k} Q_{k}(\varphi, \xi) ; M=\sum_{k=0}^{\infty} n^{-k} M_{k}(\varphi, \xi) ; \\
u=\sum_{k=0}^{\infty} n^{-k} u_{k}(\varphi, \xi) ; w=\sum_{k=0}^{\infty} n^{-k} w_{k}(\varphi, \xi),
\end{gathered}
$$

где $N_{k}, Q_{k}, M_{k}, u_{k}, w_{k}$ - периодические по $\xi$ функции с периодом $2 \pi$.

В результате приходим к уравнению

$$
M_{0}=-E I k\left(\frac{d^{2} w_{0}}{d \varphi^{2}}+w_{0}\right)
$$

где $k=\frac{\hat{a}}{s}, \hat{a}=\left(\frac{1}{2 \pi} \int_{0}^{2 \pi} a^{-1} d \xi\right)^{-1}, a(\xi)=\frac{r}{A^{2}} ; s=\frac{1}{2 \pi} \int_{0}^{2 \pi} A d \xi$.

Запишем, следуя схеме, предложенной Тимошенко для гладкого кольца [7], уравнение равновесия элемента гофрированного кольца в состоянии, отклоненном от исходного, и из решения этого уравнения найдем величину давления $q_{n}$, которая делает такую деформацию возможной. Оси симметрии «сплющенной» формы равновесия принимаем за координатные оси $x, y$ и произведем разрез кольца по горизонтальной оси симметрии (рис. 3).

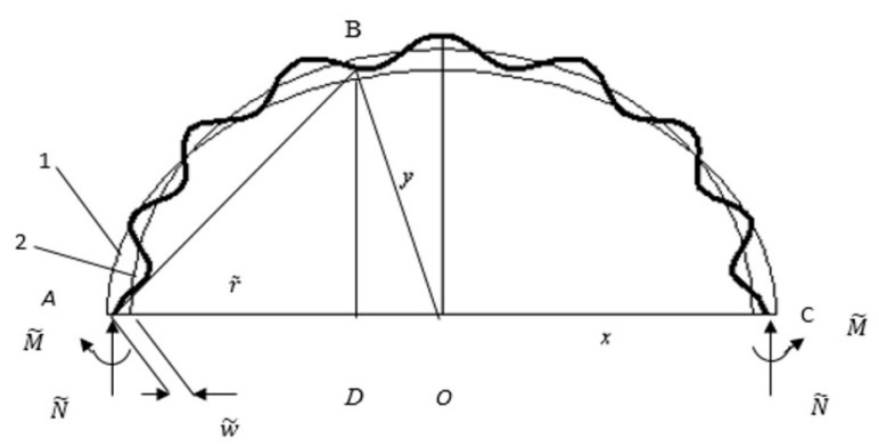

Рис. 3 - Разрез гофрированного кольца после потери устойчивости:

1,2 - базовая ось соответственно до и после потери устойчивости;

$\widetilde{\mathbf{N}}, \widetilde{\mathbf{M}}, \widetilde{\mathbf{W}}, \tilde{\mathbf{r}}$ - внутреннее усилие, момент, прогиб и радиус в точке разреза

Из рис. 3 следует, что

$$
M_{0}=\widetilde{M}_{0}-q_{n 0}\left(\widetilde{r} \widetilde{w}_{0}-R w_{0}\right) .
$$


Подставляем выражение (12) в (11) и получаем осредненное дифференциальное уравнение равновесия гофрированного кольца после «общей» потери устойчивости:

$$
\frac{d^{2} w_{0}}{d \varphi^{2}}+\beta^{2} w_{0}=\alpha
$$

где $\beta^{2}=1+\frac{q_{n 0} R}{E I k} ; \alpha=\frac{1}{E I k}\left(q_{n 0} \widetilde{r} \widetilde{w}_{0}-\widetilde{M}_{0}\right)$.

Запишем общее решение дифференциального уравнения (13):

$$
w_{0}=C_{1} \sin \beta \varphi+C_{2} \cos \beta \varphi+\frac{\alpha}{\beta^{2}},
$$

где $C_{1}, C_{2}$ - постоянные интегрирования находятся из условий симметрии

$$
\frac{d w_{0}}{d \varphi}=0 \text { при } \varphi=0, \frac{\pi}{2} .
$$

Подставляя выражение (14) в уравнение (15), находим

$$
C_{1}=0 ; \beta=2 m, m=1,2, \ldots .
$$

Полагая $m=1$, находим из (16) критическое давление общей потери устойчивости гофрированного кольца

$$
q_{n 0}=3 E I k / R .
$$

При $h=0$ из (17) получаем известную фрормулу величины критического гидростатического давления для гладкого кольца $p^{0}=3 E I / R^{3}$.

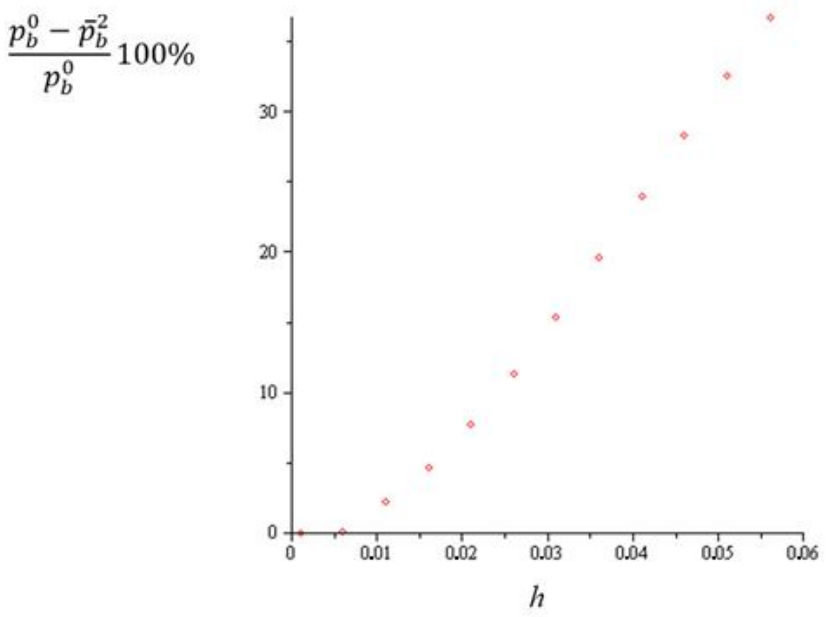

Рис. 4 - График уменьшения критического давления гофрированного кольца с косинусоидальным гофром с ростом относительной амплитуды гофра $h=H / R$ 
Таким образом, получено математическое обоснование применения эквивалентно жесткостной модели гофрированного кольца в задаче устойчивости.

На рис. 4 показано уменьшение величины критического давления гофрированного кольца $q_{n 0}$ по сравнению с гладким круговым кольцом того же радиуса для косинусоидального гофрра с ростом относительной амплитуды гофра $h=H / R$.

Выводы. Таким образом, увеличение амплитуды гофрировки уменьшает величину критического давления для гофрированного кольца. Если же сравнивать круговые и гофрированные кольца одинаковых радиуса и веса, то эта разница будет еще существеннее. Поэтому при ограничениях по весу более выгодным для повышения критического давления является увеличение поперечного сечения, чем гофрирование.

Уточнение эквивалентной жесткости (3) понижает величину критического давленичя. Эта разница увеличивается с ростом относительной амплитуды гофра $h$.

\title{
БИБЛИОГРАФИЧЕСКИЕ ССЫЛКИ
}

1. Adriaenssens S.; Dejong M. Form finding of corrugated shell structures for seismic design and validation using non-linear pushover analysis // Eng. Struct. 2019, Vol.181. P. 362-373.

2. Andreeva L.E._Elastic Elements of Instruments; Israel Program for Scientific Translations: Jerusalem, Israel, 1966. 284 p.

3. Andrianov I.I., Awrejcewicz J., Diskovsky A.A. The optimal design of a functionally graded corrugated cylindrical shell under axisymmetric loading // Int. J. Nonlinear Sci. Numer. Simul. 2019. Vol. 20. P. 387-398.

4. Andrianov I.V,; Awrejcewicz J,; Diskovsky A.A. Design optimization of FGM beam in stability problem // Eng. Comput. 2019, Vol.36. P. 248-270.

5. Donnell L.H. The flexibility of corrugated pipes under longitudinal forces and bending // Trans. ASME. 1932. Vol.54. P. 69-75

6. Timoshenko S.P., Gere J.M. Theory of Elastic Stability, 2nd ed. McGraw-Hill: New York, NY, USA, 1961. 361 p.

7. Wang K., Zhou M., Hassanein M.F., Zhong J., Ding, H., An L. Study on elastic global shear buckling of curved girders with corrugated steel webs: Theoretical analysis and FE modeling // Appl. Sci. 2018. Vol. 8. P. 2457.

УДК 539.3

\author{
О. А. Дисковський, д-р техн. наук,
}

А. А. Косиченко, канд. техн. наук,О. В. Махницький

\section{СТІЙКІСТЬ ГОФРОВАННОГО КІЛЬЦЯ ПІД ДІєЮ ЗОВНІШНЬОГО ТИСКУ}

Розглядається задача стійкості гофрованого кільця при гідростатичному тиску. Аналізуються два основні підходи до вирішення: метод еквівалентної 
жорсткості і метод асимптотичної гомогенізації. Застосування методу асимптотичної гомогенізації дозволило математично обґрунтувати і уточнити метод еквівалентної жорсткості. Проведено аналіз впливу параметрів гофрування на величину критичного тиску.

Ключові слова: стійкість; гофроване кільце; зовнішній тиск; еквівалентна жорсткість;, асимптотична гомогенізація.

Гофрування гладких оболонок широко використовується для зміни їх жорсткості. Зокрема, для циліндричних оболонок, що знаходяться під зовнішнім тиском, використовується гофрування твірної; гофрування директриси значно підвищує стійкість таких оболонок при осьовому стисненні. Тому вивчення стійкості гофрованих оболонок під зовнішнім тиском має практичне значення. Методи, що використовуються в даний час для розрахунку гофрованих пластин і оболонок, можна розділити на три групи: обчислювальні моделі, засновані на методі скінченних елементів, підхід еквівалентної жорсткості на вигин та метод асимптотичної гомогенізації. Метод скінченних елементів дозволяє розрахувати майже будь-яку гофровану оболонку $[1,7]$, але на ранніх стадіях проектування дуже корисні прості аналітичні рішення. До обчислювальної революції основним методом розрахунку гофрованих оболонок було моделювання еквівалентних жорсткостей [2, 5]. Основним недоліком цього підходу $є$ труднощі в оцінці його точності та сорери застосування. У цих роботах використовується метод асимптотичної гомогенізації [3, 4].

Постановка проблеми. Радіус гофрованого кільця в полярній системі координат становить

$$
r=R(1+h g(n \varphi))
$$

де $R$ - радіус основного кільцевого кільця; $h=\frac{H}{R}, H$ - глибина гофри; $g(n \varphi)$ - періодична функція з періодом $\frac{2 \pi}{n}$ (крок гофрування); $n$ - кількість гофрів; $0 \leq \varphi \leq 2 \pi,|h g| \leq 1 ; h \ll 1 ; n \gg 1$.

Припускаємо, що для кільця гіпотеза плоского перерізу задовольняється, а величина згинального моменту пропорційна зміні кривини середньої поверхні кільця у розглянутому перерізі. Ми маємо справу із загальною нестабільністю рифленого кільця, тобто переходом до форми 3 некруглим основним кільцем. Місцеві режими вигину відсутні. Також справедливими є такі припущення: просторові форми рівноваги не розглядаються; під час деформації навантаження залишається спрямованим до деформованого кільця нормальним, а його інтенсивність не змінюється.

Еквівалентний підхід жорсткості на вигин. Замінимо рифлене кільце круговим радіусу $R$ з еквівалентною жорсткістю на вигин. Щоб визначити цю жорсткість, виокремлюючи одну хвилю гофра, і через велику кількість таких хвиль, кривиною основного кола можна знехтувати. Ефективна жорсткість на вигин D - це жорсткість, яка забезпечує рівність 
десормацій вихідної криволінійної та апроксимаційної прямолінійних балок. В результаті отримуємо

$$
D=\epsilon E I .
$$

Профіль гофрування має дві важливі характеристики, а саме, $n \mathrm{i}$ $h$. Тиск вигинання залежить від параметра $n h$, який можна назвати параметром гофра. Для $h=0 \quad \epsilon=1$, із збільшенням $n h$ критичний тиск зменшується.

Ми використовуємо метод асимптотичної гомогенізації

$$
\frac{d^{2} w_{0}}{d \varphi^{2}}+\beta^{2} w_{0}=\alpha
$$

де $\beta^{2}=1+\frac{\bar{p}}{\hat{k}} ; \hat{k}=\frac{\dot{\hat{a}}}{\dot{s}} ; \alpha=\frac{\bar{p}}{2 \hat{a}}(\ddot{s}-s \widetilde{r}(\widetilde{r}-2 \widetilde{w}))-\frac{s \bar{M}}{\widehat{a}}$.

Загальним розв'язком рівняння (3) $€$ :

$$
w_{0}=C_{1} \sin \beta \varphi+C_{2} \cos \beta \varphi+\frac{\alpha}{\beta^{2}},
$$

де $C_{1}, C_{2}$ - константи інтегрування.

Для функції $w_{0}$ маємо:

$$
\frac{d w_{0}}{d \varphi}=0 \text { при } \varphi=0, \frac{\pi}{2} .
$$

Підставляючи вираз (4) у рівняння (5), знаходимо

$$
C_{1}=0 ; \beta=2 m, m=1,2, \ldots .
$$

Припускаючи $m=1$, з виразів (3) - (5) можна виявити тиск вигину гофрованого кільця

$$
\bar{p}_{b}^{2}=3 \hat{k} .
$$

Таким чином, отримано обґрунтування використання підходу до еквівалентної жорсткості на вигин до проблеми стійкості гофрованого кільця.

UDC 539.3

\author{
A. A. Diskovsky, Dr. Sci. (Tech.), \\ A. A. Kosichenko, PhD (Tech.), A. V. Makhnitskiy \\ BUCKLING OF CORRUGATED RING \\ UNDER EXTERNAL PRESSURE
}

Stability analysis of corrugated ring subjected to uniform external pressure is under consideration. Two main approaches to solving this problem are analyzed: equivalent bending stiffness approach and asymptotic homogenization method. In this paper, we developed an asymptotic homogenization method, which made it possible to mathematically substantiate and refine the equivalent bending stiffness approach. The influence of the corrugation parameters on the buckling pressure are analyzed. 
Keywords: stability; corrugated ring; external pressure, equivalent bending stiffness approach; asymptotic homogenization method.

Corrugation of smooth shells is widely used to change their stiffness. In particular, for cylindrical shells under external pressure, the corrugation of the generatrix is used; corrugation of the directrix significantly increases the stability of such shells under axial compression. Therefore, the study of the stability of corrugated shells under external pressure is of practical importance.

The methods currently used to calculate corrugated plates and shells can be subdivided into three groups: computational models based on finite element method, equivalent bending stiffness approach and asymptotic homogenization method. Finite element method in principle make it possible to calculate any corrugated shell $[1,7]$, but for the early stages of design, simple analytic solutions are very useful. Before computational revolution, the main method for calculating corrugated shells was equivalent stiffnesses modeling $[2,5]$. The main disadvantage of this approach is the difficulty in estimation its accuracy and area of applicability. In these papers, the asymptotic homogenization method $[3,4]$ is used.

A statement of the problem. The radius of the corrugated ring in the polar coordinate system is

$$
r=R(1+h g(n \varphi))
$$

where $R$ is the radius of the basic circular ring; $h=\frac{H}{R}, H$ is the corrugation depth, $g(n \varphi)$ is the periodic function with period $2 \pi / n$ (pitch of corrugation), $n$ is the number of corrugations, $0 \leq \varphi \leq 2 \pi,|h g| \leq 1 ; h \ll 1 ; n \gg 1$. We assume that for the ring, the hypothesis of flat cross-section is satisfied and the magnitude of the bending moment is proportional to the change in the curvature of the middle surface of the ring in the section under consideration.

We deal with the general instability of the corrugated ring, i.e. the transition to the form with non-circular basic ring. Local buckling modes are absent. The following assumptions are also valid: spatial forms of equilibrium are not considered; during deformation, the load remains directed normal to the deformed ring, and its intensity does not change.

Equivalent bending stiffness approach. Let us replace the corrugated ring with a circular one radius $R$ with the equivalent bending stiffness. To determine this stiffness, following, we single out one corrugation wave and due to the large number of such waves, the curvature of the basic circle can be neglected. Effective bending stiffness $D$ is stiffness that ensures equality of deformations of the original curvilinear and approximating rectilinear beams. As a result, one obtains

$$
D=\epsilon E I,
$$

Corrugation profile has two important characteristics, namely, $n$ and $h$. The buckling pressure depends on the parameter $n h$, which can be called the corrugation parameter. For $h=0$ one has $\epsilon=1$, with increasing $n h$ the critical pressure decreases. 
Using asymptotic homogenization method, we have

$$
\frac{d^{2} w_{0}}{d \varphi^{2}}+\beta^{2} w_{0}=\alpha,
$$

where $\beta^{2}=1+\frac{\bar{p}}{\hat{k}} ; \hat{k}=\frac{\dot{\hat{a}}}{\dot{s}} ; \alpha=\frac{\bar{p}}{2 \hat{a}}(\ddot{s}-s \widetilde{r}(\widetilde{r}-2 \widetilde{w}))-\frac{s \bar{M}}{\hat{a}}$.

The general solution of the (3) is:

$$
w_{0}=C_{1} \sin \beta \varphi+C_{2} \cos \beta \varphi+\frac{\alpha}{\beta^{2}},
$$

where $C_{1}, C_{2}$ are the integration constants.

For function $w_{0}$ one has:

$$
\frac{d w_{0}}{d \varphi}=0 \text { at } \varphi=0, \frac{\pi}{2} .
$$

Substituting expression (4) into Equations (5), we find

$$
C_{1}=0 ; \beta=2 m, m=1,2, \ldots .
$$

Assuming $m=1$, one finds from expressions (3-5) the buckling pressure of the corrugated ring

$$
\bar{p}_{b}^{2}=3 \hat{k} \text {. }
$$

Thus, the rationale for the use of the equivalent bending stiffness approach to the problem of the stability of a corrugated ring is obtained.

\section{REFERENCES}

1. Adriaenssens S.; Dejong M. Form finding of corrugated shell structures for seismic design and validation using non-linear pushover analysis // Eng. Struct. 2019, Vol.181. P. 362-373.

2. Andreeva L.E._Elastic Elements of Instruments; Israel Program for Scientific Translations: Jerusalem, Israel, 1966. 284 p.

3. Andrianov I.I., Awrejcewicz J., Diskovsky A.A. The optimal design of a functionally graded corrugated cylindrical shell under axisymmetric loading // Int. J. Nonlinear Sci. Numer. Simul. 2019. Vol. 20. P. 387-398.

4. Andrianov I.V,; Awrejcewicz J,; Diskovsky A.A. Design optimization of FGM beam in stability problem //Eng. Comput. 2019, Vol.36. P. 248-270.

5. Donnell L.H. The flexibility of corrugated pipes under longitudinal forces and bending // Trans. ASME. 1932. Vol.54. P. 69-75

6. Timoshenko S.P., Gere J.M. Theory of Elastic Stability, 2nd ed. McGraw-Hill: New York, NY, USA, 1961. $361 \mathrm{p}$.

7. Wang K., Zhou M., Hassanein M.F., Zhong J., Ding, H., An L. Study on elastic global shear buckling of curved girders with corrugated steel webs: Theoretical analysis and FE modeling // Appl. Sci. 2018. Vol. 8. P. 2457.

Днепропетровский государственый

университет внутренних дел,

Днепр,

Украина

Надійшла до редколегії 09.06.2020 